\title{
Aphonia-deafness-retinal dystrophy-bifid halluces-intellectual disability syndrome
}

INSERM

\section{Source}

INSERM. (1999). Orphanet: an online rare disease and orphan drug data base. Aphoniadeafness-retinal dystrophy-bifid halluces-intellectual disability syndrome. ORPHA:324540

Aphonia-deafness-retinal dystrophy-bifid halluces-intellectual disability syndrome is a rare, genetic, multiple congenital anomalies/dysmorphic syndrome characterized by moderate to severe intellectual disability, congenital aphonia, hearing loss, optic atrophy, retinal dystrophy, broad thumbs and duplicated halluces. Facial dysmorphism (incl. thick eyebrows, ptosis, long, downslanting palpebral fissures, microstomia, low-set, posteriorly rotated ears) and genital abnormalities are also associated. 